TERRA. Revista de Desarrollo Local e-ISSN: 2386-9968

Número 6 (2020), 331-335

DOI 10.7203/terra.6.16589

IIDL - Instituto Interuniversitario de Desarrollo Local

\title{
Reseña. Movilidades, trayectorias vitales y sostenibilidad rural
}

\author{
Lidia Espí Verdú \\ Graduada en Geografía y Medio Ambiente (Valencia, España) \\ lidia.ev96@gmail.com
}

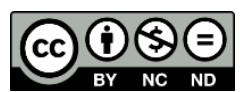

Esta obra se distribuye con la licencia Creative Commons Reconocimiento-NoComercial-SinObraDerivada 4.0 Internacional 


\section{SECCIÓN RESEÑAS}

\section{Reseña. Movilidades, trayectorias vitales y sostenibilidad rural}

Resumen: Esta obra reivindica que no se puede hablar del mundo rural como un sistema cerrado o autárquico, ya que la globalización ha permitido entenderlo como un sistema abierto donde las movilidades actuales que se dan en el entorno, potencian las conexiones entre lo urbano y lo rural, una relación cada vez menos uniforme y más acordada. Estas relaciones de movilidad son mucho más que un proceso existente en el territorio, son un hecho que ha potenciado el cambio de la ruralidad y que poseen gran importancia.

Palabras clave: Movilidades, sostenibilidad, espacios rurales, São Jacinto (Aveiro, Portugal), Gran Vega de Sevilla, montaña pirenaica navarra.

Recibido: 30 de enero de 2020

Devuelto para revisión: -

Aceptado: 30 de enero de 2020

Referencia / Citation:

Espí, L. (2020). Reseña. Movilidades, trayectorias vitales y sostenibilidad rural. TERRA. Revista de Desarrollo Local, (6), 331-335. DOI 10.7203/terra.6.16589 
Jesús Oliva Serrano (coordinador)

\section{MOVILIDADES, TRAYECTORIAS VITALES Y SOSTENIBILIDAD RURAL}

Pamplona, Universidad Pública de Navarra, 2018, 220 páginas

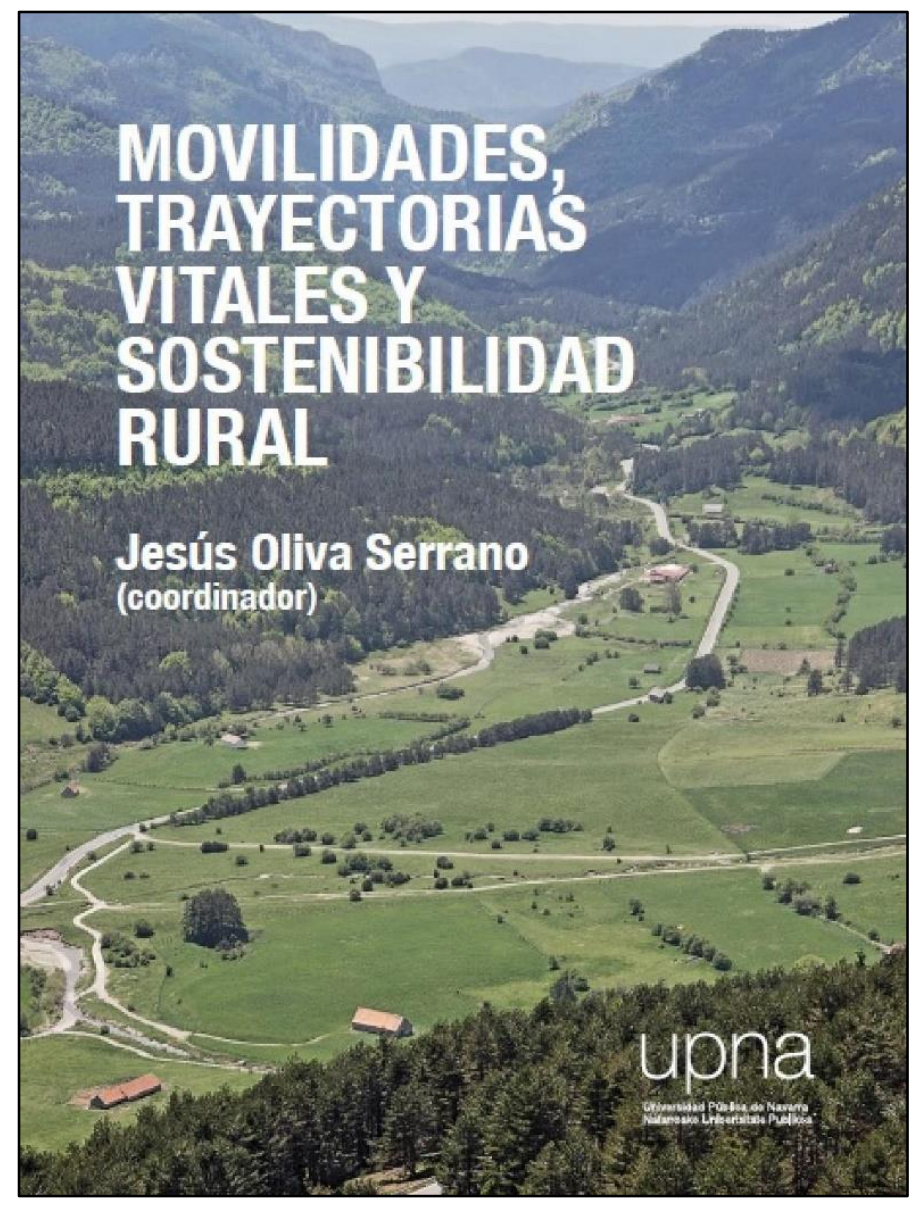

Esta obra reivindica que no se puede hablar del mundo rural como un sistema cerrado o autárquico, ya que la globalización ha permitido entenderlo como un sistema abierto donde las movilidades actuales que se dan en el entorno, potencian las conexiones entre lo urbano y lo rural, una relación cada vez menos uniforme y más acordada. Estas relaciones de movilidad son mucho más que un proceso existente en el territorio, son un hecho que ha potenciado el cambio de la ruralidad y que poseen gran importancia.

El primer capítulo del libro aborda los temas teóricos sobre las movilidades, las trayectorias vitales y la sostenibilidad rural, conceptos que en los siguientes capítulos son explicados mediante casos de estudio localizados en São Jacinto

(Aveiro, Portugal), la Gran Vega de Sevilla y la montaña pirenaica navarra. Para terminar, en el último capítulo se aportan una serie de conclusiones muy interesantes para su aplicación en investigaciones posteriores de corte similar, y como aportación a la gobernanza rural.

En los capítulos 2 y 3 se trata el caso de São Jacinto, en donde se nos explica la dificultad de definir y delimitar la ruralidad en espacios litorales que se moldean según actividades y políticas estatales dirigidas a la conservación de los espacios naturales. Con la desindrustralización y la aparición de nuevas economías de servicios, esta área portuguesa ha visto su organización socioeconómica alterada, puesto que su estructura demográfica ha cambiado, la oferta de trabajo no es suficiente y el sistema de transporte público es tan ineficiente que se ha hecho necesario crear nuevas movilidades mediante la autoorganización vecinal. Sin embargo, mientras va perdiéndose población arraigada al territorio por la complicada red de movilidades, va aumentando el turismo de temporada.

En los capítulos 4 y 5 aparece el caso de la Gran Vega de Sevilla, un claro ejemplo de la complejidad que ampara a las áreas periurbanas, y una reivindicación para visibilizar más el potencial de la movilidad como factor que favorece el arraigo en las zonas rurales. Los procesos de colonización y contraurbanización, junto con las movilidades del territorio, han transformado el modo de vida de este conjunto de población mediante procesos de 
continua reestructuración socioespacial. La idea de imponer lo urbano sobre lo rural ha sido el gran imperativo que ha determinado la planificación urbana de este espacio. La movilidad, tanto individual como colectiva, es un factor clave para la vida diaria de los vecinos/as de esta área que vio cómo se potenció su crecimiento demográfico a partir de los años noventa debido a su proximidad con el área urbana y la posibilidad de flujos de movilidad.

Para terminar con los casos de estudio, en los capítulos 6 y 7 se examina la montaña pirenaica navarra (Figura 1). Este tema de investigación nos explica cómo a los problemas típicos de los espacios rurales como, por ejemplo, la escasez de empleo local, los desequilibrios demográficos o una complicada orografía, se le suma la tendencia a la concentración y/o privatización de los servicios. Todo esto hace necesaria una intensificación en la movilidad de los vecinos/as, una movilidad que no se presenta siempre igual, sino que tiene una gran diversidad y complejidad de formas atendiendo a las estrategias de la población centradas en la movilidad y en la bi-residencialidad (por ejemplo se crean grupos de WhatsApp donde se organizan padres y madres para llevar a sus hijos a actividades extraescolares o también aumenta el número de profesionales que viven durante los días laborales en el pueblo porque tienen su trabajo ahí, pero que los fines de semana se van a sus ciudades de origen).

Figura 1. Índice de contenidos

\begin{tabular}{|c|c|c|}
\hline & Movilidades, hibridaciones rurales y paisajes sociales & $21-33$ \\
\hline & Luis Camarero 돔, Jesús Oliva Serrano 돔 & $\square$ \\
\hline & Discontinuidades de una lengua de tierra. La Freguesia de Sao Jacinto y la Región de Aveiro & $35-51$ \\
\hline & Elisabete Figueiredo, Jesús Oliva Serrano ${ }_{\square}$, Jonathan D. Teles Breda & $\square$ \\
\hline & Un puente y un espejismo. Sostenibilidad rural y movilidades en Sao Jacinto (Aveiro) & $53-72$ \\
\hline & Elisabete Figueiredo, Jesús Oliva Serrano ${ }_{\square}{ }_{a}$, Jonathan D. Teles Breda & $\square$ \\
\hline & La ruralidad periurbana en la Gran Vega de Sevilla & $73-115$ \\
\hline & Mariano Pérez-Humanes $﹎{\square}$, Carmen Guerra de Hoyos ${ }_{\square}{ }_{\square}$, Carlos Tapia Martín 돔 & 口 \\
\hline & Proyectos vitales y residenciales en la Gran Vega de Sevilla & $117-136$ \\
\hline & Manuel Tomás González Fernández ¿品, Inmaculada Montero Logroño & $\square$ \\
\hline & Ruralidad remota y monitorización a distancia. El caso de la montaña pirenaica navarra & $137-161$ \\
\hline & Andoni Iso Tinoco, Jesús Oliva Serrano $\square_{\square}$ & ㅁ \\
\hline & Estrategias sociales y movilidad en la montaña navarra & $163-178$ \\
\hline & Elvira Sanz Tolosana, Ion Martínez Lorea & $\square$ \\
\hline & Conclusiones para la investigación y la gobernanza rural & $179-192$ \\
\hline & Jesús Oliva Serrano 돔 & $\square$ \\
\hline
\end{tabular}

Fuente: https://dialnet.unirioja.es/servlet/libro?codigo=722420 (consulta realizada el 25/01/2020).

El movimiento de los grupos de población rural ha aumentado, y con ella el flujo de personas, capitales, objetos o informaciones. Los espacios rurales han visto diversificada su esfera social debido a las migraciones internacionales, los cambios residenciales, el acceso al automóvil o las nuevas tecnologías de la comunicación y el conocimiento. Tanto las movilidades como las inmovilidades rurales son existentes en estos territorios, y es que no están imitando los patrones de comportamiento contemporáneos, sino que ofrecen nuevos panoramas. La nueva migración o dinámicas residenciales han incrementado el 
cambio de la ruralidad y con ello, los límites urbano-rurales; pero el hecho de que las movilidades no sean uniformes ha destapado nuevos desequilibrios sociales $y$ vulnerabilidades.

Es esencial tener en cuenta que la accesibilidad y la movilidad están vinculadas a la calidad de vida en el mundo rural. Tener accesibilidad a determinados servicios o recursos puede ser clave para mantener la población de un territorio amenazado por la despoblación. Luego, mientras que las áreas rurales bien conectadas con los centros urbanos se ven revitalizadas, las que no lo están tanto, presentan problemas socioeconómicos graves donde se alojan los grupos sociales más marginados. Las movilidades de las que hablamos no son solamente de aspecto material, sino que también son de aspecto sociocultural, y esto provoca un cambio en el comportamiento de la población rural. La crisis del mundo rural y su correspondiente falta de determinados servicios básicos en sanidad o en educación, por ejemplo, desafía el bienestar social de sus vecinos/as. El desarrollo local o el planeamiento territorial pueden favorecer ciertas movilidades que produzcan un aumento en la calidad de vida de la población de estas áreas, y un mayor equilibrio en el territorio y sus relaciones. Estas prácticas son esenciales para luchar contra los problemas sociales actuales del mundo rural mientras lo acompañamos en su cambio.

La obra que aquí reseñamos, nos da a entender el nuevo paradigma de la movilidad, un paradigma donde los paisajes socioterritoriales no poseen un carácter replicable, y junto con la hibridación, pasan a ser dos herramientas clave de análisis para la dinamización del desarrollo local y la cohesión social del mundo rural. La parte teórica e introductoria del primer capítulo es completa y precisa, en gran medida como resultado de su abundante y contrastada citación que, en su conjunto, hace de estos primeros capítulos una aportación fundamental de conceptos para la comprensión del conjunto de la obra. Los casos de estudio son muy diversos entre sí, permitiendo al estudio ofrecer un panorama rico en matices, capaz de facilitarnos la comprensión de procesos actuales de movilidad e hibridación de los espacios rurales (tanto litorales como de interior). Todo esto nos puede llevar a imaginar cuál será su futuro, al menos a corto o medio plazo.

Para concluir nos quedaremos con una idea fuerza, capaz de sugerir nuevas investigaciones: los procesos de movilidad e hibridación pueden ser utilizados de forma integrada mediante políticas territoriales, para contribuir y reconstruir de este modo, como una vía más, la resiliencia de los espacios rurales.

Lidia Espí Verdú

Graduada en Geografía y Medio Ambiente

Universitat de València

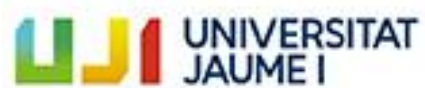

Institut Interuniversitari de Desenvolupament Local

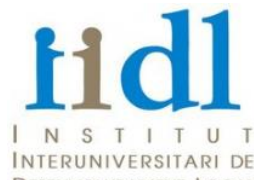
INTERUNIVERSITARI DE
DESENVOLUPAMENT LOCAL 